\title{
REPLY TO ØYSTEIN LINNEBO AND STEWART SHAPIRO
}

\section{Ian Rumfitt}

\begin{abstract}
I am very grateful to Øystein Linnebo and Stewart Shapiro for their thoughtful and penetrating essays on The Boundary Stones of Thought (henceforth BST). In publishing the book, I hoped to engage the interest of others who have thought hard about the questions it addresses. The present symposium shows that that aim was achieved.
\end{abstract}

\section{Reply to Linnebo's criticisms}

Øystein Linnebo's contribution sets me a practical as well as an intellectual challenge. In the second part of $B S T$, I tried to explain where a number of well-known arguments for deviating from classical logic go wrong. As well as criticizing my analysis of one such-due to William Tait (1998)—Linnebo advances (in his §6) an interesting new argument against using classical logic in transfinite set theory. (As he acknowledges, his argument develops some of Michael Dummett's ideas about 'indefinitely extensible concepts', but Linnebo brings in so many additional elements that the end result is new.) With limited space at my disposal, I devote this section to replying to Linnebo's criticisms of my discussion of Tait before venturing (in §2) a preliminary assessment of his new argument against classical logic. Linnebo expounds the argument to which I was responding as follows: 
(1) For classical quantification to be permissible [i.e. for it to be permissible to apply the rules of classical quantificational logic], a domain must have been categorically characterized.

(2) The domain of set theory cannot be categorically characterized.

Therefore

(3) Classical quantification is not permissible when reasoning about set theory.

As Linnebo says, I accept premiss (2). He also accepts (2) but he criticizes my objection to Vann McGee's (1997) attempt to provide a categorical axiomatization of set theory. McGee had postulated a new axiom stating that there is a set of all Urelemente, i.e. a set of all things that are not sets. Linnebo accepts my objections to this putative axiom but complains that they do not get to the heart of the matter. McGee could have achieved categoricity, he notes, by postulating instead that there are no more Urelemente than there are pure sets. My objections do not refute this weaker postulate, which is all that McGee's argument for categoricity requires.

I accept this criticism and gratefully adopt Linnebo's own counter to McGee. As Linnebo remarks, even the weaker postulate has the height of the set-theoretic hierarchy being determined by an extra-mathematical matter-viz., whether there are more non-sets than pure sets. Throughout Chapter Nine of $B S T$, though, I was exploring the consequences of the philosophy of mathematics sketched in Dummett's paper 'What is Mathematics About?' (Dummett 1993. Its key contention is that pure mathematics is determinate only insofar as our mathematical practices determine it. In particular, then, the height of the hierarchy, if it is determinate at all, must be determined by those practices: it cannot depend on the extramathematical issue of whether there are, or are not, more non-sets than pure sets. Whatever may be the intrinsic merits of the weaker postulate, all one needs to say about it in the present 
dialectic context is that it controverts the philosophical premiss on which the discussion proceeds. So premiss (2) stands.

What about premiss (1)? Somewhat oddly, Linnebo writes as though I was trying to defend it. To the contrary. My chapter was directed towards defending the use of classical quantificational logic in set theory. Since I accept (2), and since (1) and (2) jointly entail (3), I reject (1). While I contended that not every statement in the language of set theory is bivalent, I denied —and still deny—-that this contention implies (1).

It will be worth rehearsing briefly my reasons for these claims. Let $L$ be the language of second-order set theory and let $T$ be whatever axiomatization of that theory you accept. The first point to note is that, if $T$ is not categorical, the standard argument for supposing that every statement in $L$ is bivalent collapses. For what could it mean-in the context of the philosophy of mathematics which I was trying to develop - to call a statement $A$ in the language of set theory true, or false? The natural answer is this: you will count $A$ as true if and only if $A$ is a semantic consequence of $T$; and you will count $A$ as false if and only if its negation is a semantic consequence of $T$ (cf. $B S T$, pp.268, 274). If $T$ were categorical, a standard argument then shows that every sentence in $L$ is either true or false (see BST pp.2678 and 314-5). If $T$ is not categorical, however, there will be actual counterexamples to bivalence; see $B S T$, p.269.

What I found in Tait's discussion of these matters, moreover, were reasons to doubt that the generally accepted axiomatization of set theory will ever be categorical. Zermelo already showed that $\mathrm{ZFC}^{2}$ is merely quasi-categorical; he also gave reasons (which Linnebo accepts) for rejecting set theories which achieve full categoricity by truncating the settheoretic universe. We have, moreover, seen why McGee's attempt at a categorical axiomatization (whether in its original version or its weaker form) is out of bounds for present purposes. Tait further argues that, so far from attaining a categorical characterization of the universe of sets, we shall never even be able to settle on a definitive axiomatization. Mathematicians characterize that universe as 'obtained by iterating the power set operation as far as possible. But this is not a mathematical characterization; rather, it is an informal idea, 
which leads us to successively stronger precise principles of iteration...Indeed, a

mathematical characterization is precluded on the iterative conception by the fact that any mathematically definable totality $P$ of such principles would lead to a new one, namely closure under all the principles in $P$. Moreover, it is not at all clear that, as we try to develop the idea of iteration as far as possible, there is only one direction this would take us...So it does not seem possible to speak of the universe of sets as a well-defined extension' (Tait 1998, p.478).

What follows if we accept premiss (2)? Let us call a semantic theory classical if it postulates that every statement of the language to which it applies is either true or false, and gives the meanings of the sentential connectives by way of their standard truth-tables. Then one thing that follows from (2) is that we cannot interpret $L$ using a classical semantic theory. For (2) generates counterexamples to bivalence.

How, then, should we interpret $L$ ? In $B S T$, I proposed a two-stage answer. As the remarks just quoted from Tait imply, an adequate interpretation of $L$ must take into account the various possible axioms - many of them using notions of which we have, as yet, no inkling - that set theorists may be led to postulate as they say which iterations of the powerset operation are possible. 'When we affirm the truth of a proposition about the universe of all sets, we must speak of it on the basis of those operations for constructing sets that have already been accepted, while admitting that further principles might also be admitted' (Tait 1998, p.478). Accordingly, the backdrop of the proposed interpretation is the space of axiom systems for set theory which are consistent extensions of ZFC ${ }^{2}$ (see BST §9.3). We then construct a Kripke semantics for $L$ relative to this space. That is, we begin by characterizing the relation of an axiom system's forcing a formula: $s$ forces an atomic formula $A$ if and only if $s$ semantically entails $A$ (BST, p.279). Recursive clauses then extend this relation to complex formulae. So, for example, the clause for disjunction says that $s$ forces ${ }^{\lceil} A \vee B^{\rceil}$if and only if $s$ either forces $A$ or forces $B$ (ibid.). Given this clause, there will be axiom systems in the space which leave certain instances of Excluded Middle unforced. For example, where 
$M C$ is a formula in $L$ which states that there is at least one measurable cardinal, the system $\mathrm{ZFC}^{2}$ entails neither $M C$ nor its negation. Thus $\mathrm{ZFC}^{2}$ does not force ${ }^{\lceil} M C \vee \neg M C$.

At this juncture, the prospects may look bleak for a defence of classical logic in set theory. It seems that we should agree with Tait that 'it is precisely in connection with the absolute, the universe of all sets, that the ideas of constructive mathematics become compelling' (Tait 1998, p.478). Well, I agree that the semantic ideas of constructive mathematics become compelling: the account of forcing just given draws on precisely those ideas. But I deny that it follows, as Tait says it does, that 'the logic that applies to arbitrary formulas of set theory, when these are interpreted in the universe of all sets, should be constructive, not classical logic' (ibid., emphasis added).

Why does this not follow? The result would follow if the condition for a conclusion to be a logical consequence of some premisses were that any member of the space which forces all the premisses also forces the conclusion. I deny, however, that this is the correct account of consequence. It is at this point that we need to bring in the second stage of the interpretation of $L$. As I argued in Chapter 3 of $B S T$, the correct condition for $A$ to be a consequence of $X$ is that $A$ should be true at every possibility where all of $X$ are true. The members of our space of axiom systems are possibilities in the relevant sense, for they are precisifications of the vague dictum 'The universe of sets is obtained by iterating the powerset operation as far as possible'. I deny, however, that a statement in $L$ is true at a possibility (i.e. at an axiom system) only when the system forces it. Rather, we should apply a negative translation and say that $A$ is true at $s$ if and only if $s$ forces its double negation (p.288). It may then be shown that every instance of ${ }^{\lceil} A \vee \neg A^{\urcorner}$is true at every $s$, so the whole interpretation validates Excluded Middle (and, in fact, all the laws of classical logic).

Why should we accept this account of truth at a possibility? In a recent critical essay on BST, Crispin Wright objected that this interpretation merely 'saves something that looks like classical logic' (Wright 2018, 8). It may, indeed, seem that my interpretation of $L$ does not really vindicate Excluded Middle, but instead merely validates all instances of 
$\left\ulcorner_{\neg \neg(A \vee \neg A)}\right.$. Since even an intuitionistic logician accepts that schema as a logical truth, I may seem to have provided no defence of the use of classical logic in set theory.

Øystein Linnebo appreciates that this objection is misplaced. He understands that $B S T$ offers 'a detailed account of why this [negative] translation is appropriate, not merely an ad hoc trick. Indeed', he goes on, $B S T$ 'can be seen as a sustained defence of the appropriateness of the translation' (Linnebo $2018 \S 2$, p.2). I could not put it better myself, but I was somewhat disappointed that Linnebo does not try to assess that defence, which is vital to my case for using classical logic in set theory. What underlies the defence is a general point about how to specify the meanings of 'or' and 'not' in relation to possibilities that are not fully determinate. It would mischaracterize our use of 'or' to say that ${ }^{\lceil} A$ or $B$ is true at a possibility $x$ if and only if either $A$ is true at $x$ or $B$ is true at $x$. For 'Either a boy or a girl is at home' is true at the possibility of a child's being at home, even though neither disjunct is true there (cf. BST p.156). What we have to say instead is that ${ }^{\lceil} A$ or $B^{\rceil}$is true at $x$ if and only if $x$ is incompatible with any possibility which itself is incompatible with the truth of both $A$ and $B$. Assuming that ${ }^{\lceil}$not $A^{\rceil}$is true at $x$ if and only if $x$ is incompatible with the truth of $A$, this vindicates the claim that $A$ is true at $s$ if and only if $s$ forces its double negation. The proposed two-stage interpretation of $L$, then, implements these general points about the meaning of 'or' and 'not' in the particular case where the relevant possibilities are consistent extensions of $\mathrm{ZFC}^{2}$.

For these reasons, I regard as otiose the modal vindication of the use of classical logic that Linnebo offers me in the last paragraph of his $\S 4$. He quotes me as claiming that 'the semantic theories that best reflect the indeterminate height of the set-theoretic universe seem only to validate weaker than classical logics' $(B S T$, p.285). Like him, though, I agree that the semblance here is not veridical: the use of classical logic may be justified, even though we cannot apply a classical semantics to the language of set theory. It is interesting to know that Putnam's modal translation of $L$ vindicates Zermelo's set theory with a classical logic, but that would appear to be an alternative route to a destination I had already reached. 


\section{Linnebo's new argument against the use of classical logic in set theory}

One moral of $B S T$ is that there is no master defence of classical logic. Arguments purporting to show that logic to be inapplicable in certain areas must be assessed on their own merits. What should we make of Linnebo's new argument against using classical logic in set theory?

This argument has the same structure as that considered in the previous section, but it involves a new notion. Linnebo begins by contrasting two ways in which we might specify a collection of objects. 'Some specifications are intensional, in the sense that they proceed by specifying a membership criterion’ (Linnebo $2018 \S 6$, p.10). Thus I might specify the collection of people in Hall by saying that they are the fellows of the college. 'Other specifications are extensional, in the sense that the collection is specified directly via its members, not by way of any membership criterion' (ibid.). Thus I might specify the collection of people in the Wharton Room by saying that they are precisely John, Sarah, and Tom.

Linnebo's basic thought is that classical quantificational logic is applicable only when a domain admits of a purely extensional specification. But when do we have such a specification? The paradigm is when we are given a comprehensive list of the domain's members, but when it is infinite no such list is possible. Linnebo looks to plural logic to provide the requisite generalization. A list such as 'John, Sarah, and Tom' may instantiate a plural variable. 'The semantic contribution of a plural variable is purely extensional. The variable contributes its one or more values, but no intension or membership criterion' (ibid.). Linnebo then contends that a domain admits of a purely extensional specification if and only if its members are the possible values of a plural variable — for short, are a plurality. By combining this contention with the thesis that classical quantification demands an extensionally specifiable domain, we reach his argument's first premiss:

(1') For classical quantification to be permissible, the domain must admit of specification by means of a plurality. 
Linnebo also holds that

$\left(2^{\prime}\right) \quad$ The domain of set theory cannot be specified by means of a plurality.

From these premisses it follows that

(3') Classical quantification is not permissible when reasoning about set theory.

Like Dummett and Tait before him, Linnebo holds that intuitionistic logic may be used in reasoning about the universe of all sets.

Linnebo's argument is clearly valid. I am less confident than he is, however, that premiss $\left(2^{\prime}\right)$ is true.

There is, in fact, an air of paradox about $\left(2^{\prime}\right)$. Given the resources of plural logic, one would have thought that it was easy to specify the universe of set theory: it comprises all and only the sets. This objection is, of course, too quick. In the paper 'Pluralities and Sets' (2010) to which he refers us, Linnebo shows himself to be well aware that he will have to restrict the Comprehension Scheme of plural logic. So, even though 'is a set' is a meaningful and non-empty predicate, Linnebo's version of plural logic offers no guarantee that there is such a plurality as the sets. All the same, since it requires restricting plural Comprehension, we surely need a good argument for $\left(2^{\prime}\right)$. What is that argument?

It runs as follows:

When a collection is specified in a purely extensional way, we can make good conceptual and mathematical sense of this collection defining a set. After all, we know exactly what the members of the would-be set are. And since the members are specified in a purely extensional way, no contradiction will ensue from positing or 
'forming' a set with precisely those members - if need be, outside of the pure extension with which we began. Since the target collection is specified in a purely extensional way, we have no problem conceiving of a richer situation in which this target collection defines a set. It would therefore be arbitrary and restrictive to deny the existence of the would-be set (Linnebo $2018 \S 6$, p.11).

In his 2010 paper, Linnebo expands on this theme of arbitrariness. The only principle for denying the existence of the would-be set, he says, is limitation of size - the idea that some collections are too large to form sets. But no non-arbitrary limit to the size of collections which can form sets has ever been found. Wherever we have a collection, then, we also have a potential set. But—in standard set theory at least—-there can be no set of all sets. So there is no collection of all sets. Awkward as its implications may be for the formalization of plural logic, premiss (2') must be accepted.

The idea that some collections are too large to form sets entranced some of the preeminent set theorists of the twentieth century. For all that, I share Linnebo's scepticism about its explanatory value. Even if we set it completely aside, however, there is a distinct idea, far more securely rooted in the predominant conception of sets, which sometimes does stop us from 'conceiving of a richer situation in which a target collection defines a set'.

At the start of 'Pluralities and Sets', Linnebo explains that by 'set' he means 'set as on the standard iterative conception, according to which sets are "formed" in stages' (2010, p.144). That is what I mean, too, but the metaphor of formation in stages implies a limitation on which collections can form sets. Under the iterative conception, a set exists only if it is formed at a certain stage. That is to say, each set must possess a rank. This means that a collection will form a set only if it is limited, not in size, but in rank: there must be an upper bound on its members' ranks if it is to form a set. There is, then, nothing 'arbitrary and restrictive' about denying that some collections form sets. To the contrary: such a restriction is implicit in the very idea of the iterative hierarchy. What is more, the collection of all sets is a paradigm of something to which this restriction applies. There is no upper bound on the 
ranks of sets. So we can explain why there is no set of all sets even though there is such a plurality as the sets. The universe of sets can be specified by means of that plurality—viz., as the domain which comprises precisely the sets—so premiss $\left(2^{\prime}\right)$ is false.

This argument against premiss $\left(2^{\prime}\right)$, moreover, does not involve any illicit switch between intensional and extensional specifications. A set's rank is determined by the ranks of its members. A purely extensional specification of the set, then, will determine that rank. So the present rationale for denying that every collection forms a set applies even when every collection and every set is specified purely extensionally.

Because I hold that $\left(2^{\prime}\right)$ is false, I judge that Linnebo's new argument against classical logic fails. It may be worth adding, though, a couple of comments about premiss (1').

Linnebo bases $\left(1^{\prime}\right)$ 'on the idea that classical quantification rests on an instance-based conception of generality' (Linnebo 2018, p.12). Some version of this idea may well be right, but one has to be careful in formulating it. In his note 15, Linnebo quotes Dummett (1981, p.530) who characterizes the classical interpretation of the quantifiers 'as infinitary conjunction or disjunction'. This is surely too strong. Frege interpreted the quantifiers classically, but he also held that 'if I utter a sentence with the grammatical subject "all men", I do not wish to say something about some Central African chief wholly unknown to me. It is thus utterly false that I am in any way designating this chief when I use the word "man"” (Frege 1895, p.454). However, if 'All men are mortal' really were an abbreviation of the indefinitely long conjunction 'Adam is mortal and Abel is mortal and...', then in uttering that sentence one would be designating each and every man.

Second, while Linnebo accepts (1'), he rejects the corresponding premiss about intuitionistic logic. For, if the corresponding premiss held, we could apply the same argument to show that intuitionistic logic was inapplicable when reasoning about all sets, contrary to Linnebo's claim that it may legitimately be applied. However, it is not at all clear what, on his account, legitimizes the use of that logic. In intuitionistic as much as in classical quantificational logic, we are expected to specify the domain —or the intuitionistic species- 
over which the variables are to range. But how, on Linnebo's account, are we to do this? As we have seen, he denies that there is any such plurality as the sets, so we cannot say that the variables are to range over all and only the sets. But then it is not clear what else one could say.

It is instructive to compare Linnebo's position here with Russell's in 'Mathematical Logic as Based on the Theory of Types' (Russell 1908). Russell's solution to the Burali-Forti Paradox was that 'we cannot say that the whole series [of ordinals] is well ordered, because the "whole series" is a fiction' (Russell 1908, p.101). That is, there is no such plurality as the ordinals (in their standard ordering) - although there are of course ordinals. But Russell held that it follows from this thesis that "“all ordinals" is an illegitimate notion'-i.e. that statements about 'all ordinals' (and about 'every ordinal') are nonsense (1908, p.63). When we wish to make generalizations about ordinals, we have to resort to claims in the form 'Any ordinal...', as when we say 'Any ordinal is either zero, a successor, or a limit'. Russell's distinction between 'all' and 'any' corresponds to the difference between ' $\forall x \varphi x$ ', which expresses a single universal proposition, and the purely schematic formula ' $\varphi x$ '. When ' $x$ ' is understood to be a free variable ranging over ordinals, this latter formula expresses no single proposition. Rather, it evinces an open-ended willingness to attribute the property of being $\varphi$ to any object that gets counted as an ordinal. Now the intuitionist, just as much as the classicist, understands ' $\forall x \varphi x$ ' to express a single proposition: the intuitionist equally allows this formula to be negated and combined with other complete formulae using sentential connectives. Given his denial that there is any such plurality as the sets, it is a nice question for Linnebo how he is going to justify regimenting general claims about sets in the properly quantified form which intuitionistic logic requires, rather than as Russellian schemata.

My last points about Linnebo's argument are implicit in the defence of my own position in the previous section. Even if Linnebo can vindicate applying an intuitionistic semantic theory to the language of set theory, it would appear still to be open to defend the use of classical logic, when reasoning about sets, by way of the negative translation of $B S T$ 
§9.5. Finally, I am uncertain whether the modal apparatus which Linnebo uses in his account really coheres with the Dummettian philosophical premiss that was the foundation of the discussion in BST Chapter Nine. Prima facie, to appeal to assumptions about which settheoretic constructions are mathematically possible is to make an appeal to facts, or putative facts, of the sort that Dummett was concerned to eschew. At the very least, we need an argument to show that the modal facts in question are implicitly determined by our actual mathematical practices.

\section{Reply to Stewart Shapiro}

Stewart Shapiro's criticisms of BST take a rather different form. Instead of analysing in detail one of my arguments, and explaining where he thinks it goes wrong, Shapiro notes a range of points in the philosophy of logic where he and I diverge. In the space at my disposal, an attempt to answer all, or even many, of these points could only be superficial. For that reason, I shall confine my reply to his observations about intuitionistic theories in $\S \S 4$ and 5 of his paper.

Quine held that adherents of rival logics perforce attach different senses to the connectives: 'here, evidently, is the deviant logician's predicament: when he tries to deny the doctrine he only changes the subject' (Quine 1986, p.81). Dummett thought the same: 'our fundamental logical laws are those which it is an essential part of our practice in speaking the language to observe. The view that revision of them involves a change in the meanings of the logical constants is unshakable' (Dummett 1991, p.302). Dummett notwithstanding, that view is one that $B S T$ tried to shake. Quine does not give an argument for his claim, and in $B S T \S 7.5$ I tried to show where Dummett's argument goes wrong.

I stand by what I said there: disputes over logical laws do not necessarily involve the disputants attaching different senses to the connectives or quantifiers. Everyone must acknowledge, though, that this is one way in which a dispute - or the appearance of one- 
may arise. In my view, the disagreement between the classical and intuitionistic versions of analysis is such a case. Since Shapiro raises the topic, I shall begin my reply to him by giving my reasons for this view.

The disagreement in question has its roots in rival metaphysical views about infinity. Intuitionists define a real number generator to be a Cauchy sequence of rationals $\left\langle r_{n}\right\rangle$ with the property that $\forall k \exists n \forall m_{m>n}\left|r_{m}-r_{n}\right|<2^{-k}$. Two real number generators $\left\langle r_{n}\right\rangle$ and $\left\langle s_{n}\right\rangle$ are said to be equivalent when $\forall k \exists n \forall m_{m>n}\left|r_{m}-s_{m}\right|<2^{-k}$. Real numbers are then identified with equivalence classes (i.e. equivalence species) of real number generators. Familiar as these notions may be from classical analysis, there is a far-reaching difference in how the two schools conceive of a Cauchy sequence. The classicist thinks of this as a completed infinity, a conception which the intuitionist rejects as incoherent. Since, for the intuitionist, any infinity is merely potential, a Cauchy sequence can be apprehended only by a way of the process whereby successtive terms are determined.

As Shapiro observes, a theorem of intuitionistic analysis is $\left.\neg^{\top} \forall x(x=0 \vee \neg x=0)\right\urcorner$. This is a flat-out denial of a validity of classical quantification theory. The theorem derives from the way intuitionists spell out the relevant notion of a process. The intuitionist's definition of a real number already ensures that there are 'weak counterexamples' to the classical law $\ulcorner\forall x(x=0 \vee \neg x=0)\urcorner$. That is, the definition already ensures that there are real numbers $r$ of which an intuitionist cannot assert $\left.{ }^{\lceil} r=0 \vee \neg r=0\right\urcorner$. For example, let $A(n)$ be a predicate of natural numbers meaning 'if $n>1$, then $2 n$ is the sum of two primes', and let $\left\langle r_{n}\right\rangle$ be the Cauchy sequence defined by

$$
r_{\mathrm{n}}= \begin{cases}2^{-n} & \text { if } \forall m \leq n A(m) \\ 2^{-m} & \text { if } \neg A(m) \wedge m \leq n \wedge \forall k<m A(k) .\end{cases}
$$

Since $A(n)$ is a decidable predicate on natural numbers, $\left\langle r_{n}\right\rangle$ is a legitimate real number generator. However, the statement ${ }^{\lceil} r=0{ }^{\rceil}$is equivalent to ${ }^{\lceil} \forall n A(n)^{\urcorner}$, i.e. to Goldbach's Conjecture $(G C)$. Since no one has a proof of Goldbach's Conjecture, nor a refutation of it, 
an intuitionist will say that we are not in a position to assert ${ }^{\lceil} G C \vee \neg G C$. Since ${ }^{\lceil} r=0{ }^{\urcorner}$is equivalent to $G C$, it follows that we are not in a position to assert ${ }^{\lceil} r=0 \vee \neg r=0{ }^{\urcorner}$either.

Stronger assumptions are needed to derive theorems— such as $\left.^{\lceil} \neg \forall x(x=0 \vee \neg x=0)\right\rceil$ which actually contradict classical validities. These stronger assumptions concern continuity. Some constructivists hold that a Cauchy sequence is a real number generator only if there is a computable rule for determining the $n^{\text {th }}$ term. Brouwer realised that this approach would yield only a 'poor and anaemic' Ersatz for classical analysis, and in his 'Second Act of Intuitionism' he postulated 'freely proceeding infinite sequences of mathematical entities previously acquired' (such as rational numbers) (Brouwer 1981, p.8). It is these 'free choice' Cauchy sequences which ensure a rich intuitionistic continuum, and it is the axioms describing their behaviour which yield the theorems of intuitionistic analysis that contradict classical validities. The axioms come in various strengths, but even the weakest, the so-called Local Continuity Principle, suffices to yield $\left.{ }^{\ulcorner} \neg \forall x(x=0 \vee \neg x=0)\right\rceil$. Where ' $n$ ' and ' $m$ ' range over natural numbers, where ' $\alpha$ ' and ' $\beta$ ' range over free choice sequences, and where ' $\beta \in$ $\bar{\alpha}(m)$ ' means 'the first $m$ elements of $\alpha$ and $\beta$ are equal', this Principle says

$$
\forall \alpha \exists n C(\alpha, n) \rightarrow \forall \alpha \exists n \exists m \forall \beta_{\beta \in \bar{\alpha}(m)} C(\beta, n)
$$

(see Dummett 2000, p.60).

It is when we ask how adherents of intuitionist analysis try to justify the Local Continuity Principle that we see clearly that they must be attaching different senses to the connectives than do classical mathematicians. Unsurprisingly, in view of its remarkable implications, even the Local Continuity Principle is hard to justify in full generality. (For discussion, see Troelstra 1977, Dummett 2000 §7.5, and van Atten and van Dalen 2002.) However, all intuitionists accept the Principle in the special case where ' $\alpha$ ' ranges only over 'lawless' choice sequences. Here the justification is straightforward. The intuitionist asks in what conditions the antecedent of the Local Continuity Principle could be proved. Because 
the sequences $\alpha$ are lawless, we shall be entitled to assert $\forall \alpha \exists n C(\alpha, n)$ only when we can be sure that the condition $\exists n C(\alpha, n)$ is met for an arbitrary lawless sequence. Since the sequence is lawless, we can be sure of this only when $C(\alpha, n)$ holds for some initial finite segment of $\alpha$. But in that case the condition $\exists n \exists m \forall \beta_{\beta \in \bar{\alpha}(m)} C(\beta, n)$ is met for arbitrary $\alpha$, so we are entitled to assert the Principle's consequent.

What is immediately relevant about this argument is what it assumes about the meaning of the conditional. It is assumed that ${ }^{\lceil} A \rightarrow B$ is assertible when, and only when, any proof of $A$ may be extended to yield a proof of $B$. The assumption is unsurprising: this is exactly how Heyting explained the meaning that intuitionists attach to ' $\rightarrow$ '. In $\S 5.2$ of $B S T$, however, I argued at length that Heyting's explanations of the connectives enforce a radical deviation from the way that ordinary speakers - and, more particularly, classical mathematicians — actually use them. In this case, then, Quine is right: in denying the doctrine of $\left.{ }^{\lceil} \forall x(x=0 \vee \neg x=0)\right)^{\natural}$, the intuitionistic analyst is changing the subject. ${ }^{1}$ /

Furthermore, I also argued in that section that Heyting's explanations do not, in the end, succeed in endowing the connectives with a coherent sense.

Does this imply that intuitionistic analysis is simply nonsensical? Not necessarily. In his paper 'An Interpretation of the Intuitionistic Sentential Calculus', Gödel observed that one can interpret Heyting's sentential calculus by means of the notions of the ordinary [i.e. the classical] sentential calculus and the notion ' $p$ is provable' (written $\mathscr{B} p$ ) if one adopts for that notion the following system $\mathfrak{I}$ of axioms:

1. $\mathscr{B} p \rightarrow p$,

\footnotetext{
${ }^{1}$ Heyting would have agreed. 'CLASSICIST: It seems to me that the difficulty is caused by your interpretation of negation, which diverges from the usual one... INTUITIONIST: I can adhere to this view...[W]e must well distinguish the use of "not" in mathematics from that in explanations which are not mathematical, but are expressed in ordinary language' (Heyting 1951, p.18).
} 


$$
\begin{aligned}
& \text { 2. } \mathscr{B} p \rightarrow . \mathscr{B}(p \rightarrow q) \rightarrow \mathscr{B} q, \\
& \text { 3. } \mathscr{B} p \rightarrow \mathscr{B} \mathscr{B} p,
\end{aligned}
$$

[along with the rule of proof: given $\vdash A$, deduce $\vdash \mathscr{B} A$ ] and translates Heyting's primitive notions as follows:

$\begin{array}{ll}\neg p & \sim \mathscr{B} p \\ p \supset q & \mathscr{B} p \rightarrow \mathscr{B} q \\ p \vee q & \mathscr{B} p \vee \mathscr{B} q \\ p \wedge q & p \cdot q\end{array}$

(Gödel 1933, p.39). I / Rasiowa and Sikorski (1953) extended this translation to the language of the predicate calculus, and Gödel's treatment of the conditional is exactly what is needed to make sense of the intuitionist's argument for the Local Continuity Principle. So we can, perhaps, make sense of the adherent of intuitionistic analysis. However, if we make sense of him by applying a non-homophonic interpretation, then what he asserts when he writes down ${ }^{\ulcorner} \neg \forall x(x=0 \vee \neg x=0)^{\urcorner}$will not contradict what a classical mathematician asserts when she writes $\lceil\forall x(x=0 \vee \neg x=0)$.

What follows from this? Stewart Shapiro writes: 'For Rumfitt, Logic, properly so called, is a "second-order" discipline that "sets forth the laws of the laws of implication. If this Logic is classical, as Rumfitt argues, then classical logic applies to any and all implication relations. But if classical logic is imposed on [the intuitionistic version of Church's Thesis], intuitionistic analysis, or smooth infinitesimal analysis..., one can derive a

\footnotetext{
2 Here, 'provable' means 'provable by some correct method', not 'provable in a given formal system $S$ '. As Gödel observes at the end of his paper, if $S$ contains $\mathfrak{I}$, then $\mathscr{B}(\mathscr{B} p \rightarrow p)$ is a theorem of $S$. If $S$ also contains arithmetic, $\mathscr{B}(\mathscr{B}(0 \neq 0) \rightarrow 0 \neq 0)$, i.e. $\mathscr{B} \neg \mathscr{B}(0 \neq 0)$, would be a theorem of $S$. However, if $\mathscr{B}$ means 'is provable in $S$ ', then by Gödel's own Second Incompleteness Theorem $\mathscr{B} \neg \mathscr{B}(0 \neq 0)$ is a theorem of $S$ only if $S$ is inconsistent.
} 
contradiction and, thus, one can derive any formula whatsoever. So, by Rumfitt's lights, either Logic, properly so-called is not classical, or else all three of the above theories are, at root, incoherent' (Shapiro 2018, p.12). It will be apparent that this is not quite right. We can make sense of intuitionistic analysis, but only via a non-homophonic translation into a language whose logic is classical. Classical logic, then, still regulates the theory's implication relation, albeit at one remove. Thus the case of intuitionistic analysis, when fully thought through, does not pose any threat to my claim that classical logic sets forth the laws of the laws of implication. Had I more space, I would make the corresponding argument for the case of intuitionistic version of Church's Thesis as well.

Smooth Infinitesimal Analysis (SIA), I readily admit, requires a different treatment. As Shapiro reminds us, and as I noted on p.214 of BST, it also contains ${ }^{\ulcorner} \neg \forall x(x=0 \vee \neg x=0)^{\urcorner}$ as a theorem. However, when we consider how that is derived from the theory's axioms, it becomes evident that we cannot dissolve the appearance of inconsistency with a classical law in the way we did with intuitionistic analysis.

SIA aspires to make explicit the conception of infinitesimals that guided the pioneers of the differential and integral calculus. One of those pioneers, De L'Hôpital, postulated that 'a curved line may be regarded as made up of infinitely small straight line segments'. This yields the first axiom of SIA

The Principle of Microstraightness: For any smooth curve $C$ and any point $P$ on $C$, there is a non-degenerate segment of $C$ (i.e. a segment of $C$ which does not reduce to a single point) which is straight around $P$; that is, $C$ is 'microstraight' around $P$.

If we apply this Principle to the parabola $y=x^{2}$ at the origin, we infer that there is a nondegenerate set $\Delta$ of magnitudes $\varepsilon$ such that $\varepsilon^{2}=0$. That is, there is a non-degenerate set of nilsquare infinitesimals. These nilsquares are elements of the 'smooth' real number-line or continuum, $R$. We are not to think of $R$ as a set of points. Rather, we are to conceive of it as 
Euclid conceived of the continuum—namely, as a nest of lines, items with length but no breadth. SIA then postulates that there are operations + and . on $R$, and a relation $<$, such that $R$ constitutes an ordered field. In particular, it postulates that any quantity in $R$ that is not identical to zero has an inverse under the . operation, i.e. a reciprocal.

We can then show that any nilsquare infinitesimal is smaller than any 'regular' (i.e. finite) real number:

Proof: Since multiplication is associative, $(\varepsilon \cdot a)^{2}=\varepsilon^{2} \cdot a^{2}$, so that $(\varepsilon \cdot a)^{2}=0$ whenever $\varepsilon^{2}=0$. Thus $\varepsilon . a \in \Delta$ whenever $\varepsilon \in \Delta$. Thus $\varepsilon . a<1$ for all $\varepsilon \in \Delta$ and any regular real number $a$. If $a$ is not identical with zero, it has a reciprocal, so for any $a \neq 0, \varepsilon<1 / a$. Given any regular positive real number $r$, no matter how small, we can always find a non-zero real number $a$ for which $1 / a<r$. So any infinitesimal is smaller than any regular real number.

By contrast, if we try to say something about the relationship between one of these nilsquares and zero - or about the relationship between one nilsquare and another-we run into trouble. Where $\varepsilon$ is an arbitrary nilsquare infinitesimal, consider the hypothesis $\left.{ }^{\lceil} \neg \varepsilon=0\right\urcorner$. In SIA, this yields a contradiction. From the hypothesis, we infer that $\varepsilon$ has a reciprocal, i.e. there exists a $y$ such that $\varepsilon . y=1$. But then $0=0 . y=\varepsilon^{2} . y=\varepsilon .(\varepsilon . y)=\varepsilon .1=\varepsilon$, contradicting $\left\ulcorner_{\neg \mathcal{E}=0)}\right.$. Now consider the hypothesis $\left.{ }^{\lceil} \forall x(x=0 \vee \neg x=0)\right\urcorner$. This entails $\left.{ }^{\lceil} \forall \varepsilon \in \Delta(\varepsilon=0 \vee \neg \varepsilon=0)\right\urcorner$. Since $\ulcorner\neg \varepsilon=0\urcorner$ is contradictory, this implies $\left\lceil^{\lceil} \forall \varepsilon \in \Delta(\varepsilon=0)^{\rceil}\right.$in both intuitionistic and classical logic. However, the axioms of SIA also entail ${ }^{\lceil} \neg \forall \varepsilon \in \Delta(\varepsilon=0)^{\urcorner}$(for a proof, see BST p.213). Since the hypothesis $\left\lceil\forall x(x=0 \vee \neg x=0)^{\urcorner}\right.$entails a contradiction, we conclude that $\ulcorner\neg \forall x(x=0 \vee \neg x=0))^{\top}$ is a theorem of SIA.

The constructivist ideas which animate intuitionistic analysis - and which enable us to make classical sense of that theory, by way of Gödel's translation - find no purchase here. Given a construction of a nilsquare infinitesimal distinct from zero, one could infer 
$\Gamma \exists \varepsilon \in \Delta \neg(\varepsilon=0)\urcorner$. However, ${ }^{\ulcorner} \neg \exists \varepsilon \in \Delta \neg(\varepsilon=0)^{\urcorner}$is a theorem of SIA. Shapiro and I agree that the logically deviant features of SIA arise, rather, from the fact that infinitesimals do not stand in a determinate order relation to zero: we cannot say that an infinitesimal is either less than zero, identical with zero, or greater than zero. Similarly, given infinitesimals $\varepsilon$ and $\delta$, we cannot say that either $\varepsilon$ is less than $\delta$, or $\varepsilon$ is identical with $\delta$, or $\varepsilon$ is greater than $\delta$. Any attempt to place them in such relations leads straight to contradiction.

At the end of his essay, Shapiro writes: 'perhaps Rumfitt is not settled on whether these theories are legitimate' (Shapiro 2018, p.18). I hope I have made it clear where I stand on intuitionistic analysis, but when it comes to SIA, I am indeed unsettled. I may have erred in giving $B S T \S 7.4$ the title 'A consolation prize for the intuitionist: Smooth Infinitesimal Analysis'. Talk of a prize does suggest that I was committed to SIA's coherence. It would have been better to call it 'Smooth Infinitesimal Analysis: a last straw for the intuitionist to clutch at?' I discussed SIA in the book because it seemed to me then-as it seems to me today - to pose an interesting challenge to the sovereignty of classical logic, one that raises different issues from the more familiar constructivist challenges. But I remain uncertain whether SIA really makes sense. It seems to capture whatever it was that the pioneers of the calculus thought that they were getting at. But it was not only the mathematically unsophisticated Bishop Berkeley who derided infinitesimals as 'ghosts of departed quantities'; Russell also dismissed them as 'unnecessary, erroneous and self-contradictory'. In any case, 'ghosts' is an apt term for quantities whose order relationship with zero, and with one another, is shrouded in impenetrable mystery. 


\section{REFERENCES}

Atten, M. van and D. van Dalen. 2002. 'Arguments for the continuity principle'. Bulletin of Symbolic Logic 8: 329-74.

Brouwer, L.E.J. 1981. Cambridge Lectures on Intuitionism, ed. D. van Dalen. Cambridge: Cambridge University Press.

Dummett, M.A.E. 1981. Frege: Philosophy of Language, $2^{\text {nd }}$ edition. London: Duckworth.

— 1991. The Logical Basis of Metaphysics. London: Duckworth.

— 1993. 'What is mathematics about?' In The Seas of Language (Oxford: Oxford University Press), pp.429-55.

- 2000. Elements of Intuitionism, $2^{\text {nd }}$ ed. Oxford: Clarendon Press.

Frege, G. 1895. 'Kritische Beleuchtung einiger Punkte in E. Schröders Vorlesungen über die Algebra der Logik'. Archiv für Systematische Philosophie I: 433-56.

Gödel, K. 1933. 'Eine Interpretation des intuitionistischen Aussagenkalküls'. Ergebnisse eines mathematischen Kolloquiums 4: 39-40.

Heyting, A. 1956. Intuitionism: An Introduction. Amsterdam: North Holland.

Linnebo, Ø. 2010. 'Sets and pluralities'. The Journal of Philosophy 107: 144-64.

— 2018. 'Rumfitt on the logic of set theory'. Inquiry:

McGee, V. 1997. 'How we learn mathematical language'. The Philosophical Review 106: $36-68$. 
Quine, W.V. 1986. Philosophy of Logic, $2^{\text {nd }}$ ed. Cambridge, Mass.: Harvard University Press.

Rasiowa, H. and R. Sikorski. 1953. 'Algebraic treatment of the notion of satisfiability'. Fundamenta Mathematicae 40: 62-95.

Rumfitt, I. 2015. The Boundary Stones of Thought: An Essay in the Philosophy of Logic. Oxford: Clarendon Press.

Russell, B.A.W. 1908. 'Mathematical logic as based on the theory of types'. American Journal of Mathematics 30: 222-62. Page references are to the reprint in Russell, ed. R. Marsh, Logic and Knowledge (London: George Allen \& Unwin, 1956), pp.59-102.

Shapiro, S. 2018. 'Possibilities, models, and intuitionistic logic: Ian Rumfitt's The Boundary Stones of Thought'. Inquiry:

Tait, W.W. 1998. 'Zermelo's conception of set theory and reflection principles'. In M. Schirn, ed., The Philosophy of Mathematics Today (Oxford: Clarendon Press), pp.469-83.

Troesltra, A.S. 1977. Choice Sequences: A Chapter of Intuitionistic Mathematics. Oxford: Clarendon Press.

Wright, C.J.G. 2018. 'How high the sky? Rumfitt on the (putative) indeterminacy of the settheoretic universe'. Philosophical Studies 175: 2067-78. 